\title{
STRONGLY HOMOGENEOUS TORSION FREE ABELIAN GROUPS OF FINITE RANK
}

\author{
DAVID M. ARNOLD ${ }^{1}$
}

\begin{abstract}
ABSTRACr. An abelian group is strongly homogeneous if for any two pure rank 1 subgroups there is an automorphism sending one onto the other. Finite rank torsion free strongly homogeneous groups are characterized as the tensor product of certain subrings of algebraic number fields with finite direct sums of isomorphic subgroups of $Q$, the additive group of rationals. If $G$ is a finite direct sum of finite rank torsion free strongly homogeneous groups, then any two decompositions of $G$ into a direct sum of indecomposable subgroups are equivalent.
\end{abstract}

D. K. Harrison, in an unpublished note, defined a $p$-special group to be a strongly homogeneous group such that $G / p G \simeq Z / p Z$ for some prime $p$ and $q G=G$ for all primes $q \neq p$ and characterized these groups as the additive groups of certain valuation rings in algebraic number fields. Richman [7] provided a global version of this result. Call $G$ special if $G$ is strongly homogeneous, $G / p G=0$ or $Z / p Z$ for all primes $p$, and $G$ contains a pure rank 1 subgroup isomorphic to a subring of $Q$. Special groups are then characterized as additive subgroups of the intersection of certain valuation rings in an algebraic number field (also see Murley [5]). Strongly homogeneous groups of rank 2 are characterized in [2]. All of the above-mentioned characterizations can be derived from the more general (notation and terminology are as in Fuchs [3]):

THEOREM 1. Let $G$ be a torsion free abelian group of finite rank. Then $G$ is strongly homogeneous iff $G \simeq R \otimes_{Z} H$ where $H$ is a finite direct sum of isomorphic torsion free abelian groups of rank $1, R$ is a subring of an algebraic number field $K$ (with $1_{K} \in R$ ), and every element of $R$ is an integral multiple of $a$ unit in $R$.

Proof. $(\Leftarrow)$ First of all, the additive group of $R$, denoted by $R^{+}$, is strongly homogeneous. Let $X$ and $Y$ be pure rank 1 subgroups of $R^{+}$. There are units $u$ and $v$ of $R$ in $X$ and $Y$, respectively. Left multiplication by $v u^{-1}$ induces an automorphism $g$ of $R^{+}$with $g(X)=Y$.

Secondly, $R \otimes_{Z} A$ is strongly homogeneous, where $A$ is a torsion free abelian group of rank 1 with $H \simeq \sum_{i=1}^{k} \oplus A$. Choose $0 \neq a \in A$ and define $\delta: R^{+} \rightarrow R \otimes_{Z} A$ by $\delta(r)=r \otimes a$. Then $\delta$ is a monomorphism. Let $X$ and $Y$

Received by the editors January 20, 1975.

AMS (MOS) subject classifications (1970). Primary 20K 15 .

Key words and phrases. Strongly homogeneous, torsion free abelian group, subrings of algebraic number fields, Krull-Schmidt property.

${ }^{1}$ Research supported in part by N.S.F. Grant GP-40872. 
be pure rank 1 subgroups of $R \otimes_{Z} A$, and define $X^{\prime}=$ (image $\delta$ ) $\cap X$ and $Y^{\prime}=$ (image $\left.\delta\right) \cap Y$. By the preceding remarks there is a unit $u \in R$ such that left multiplication by $u$ induces an automorphism $g$ of image $\delta$ and $g\left(X^{\prime}\right)=Y^{\prime}$. Therefore, left multiplication by $u$ induces an automorphism $h$ of $R \otimes_{Z} A$ extending $g$. Since $\operatorname{rank}(A)=1,\left(R \otimes_{Z} A\right)$ /image $\delta$ is torsion. Consequently, $h(X) \subseteq Y$, for if $x \in X$, then $m x \in X^{\prime}$ for some $0 \neq m \in Z$; $m h(x)=h(m x)=g(m x) \in Y^{\prime} \subseteq Y$; and $Y$ is pure in $R \otimes_{Z} A$. Thus $h(X)$ $=Y$ since $h$ is an automorphism.

Finally, $G=R \otimes_{Z} H$ is strongly homogeneous. Note that $R$ is a principal ideal domain since every ideal of $R$ is of the form $n R$ for some $n \in Z$ (since $R$ is a subring of an algebraic number field; every ideal is finitely generated; and every element of $R$ is an integral multiple of a unit in $R$ ). Also, $R \otimes_{Z} A$ is an $R$-module of $R$-rank 1 so that $G$ may be regarded as an $R$-direct sum of isomorphic torsion free $R$-modules of $R$-rank 1. Consequently, every $R$-pure submodule of $G$ is a direct summand of $G$ (see Fuchs [3, p. 115] for the case that $R=Z$ and observe that the same proof is true in this case).

Suppose that $X$ is a pure rank 1 subgroup of $G$ and let $B$ be the pure subgroup of $G$ generated by $R X$. Then $B$ is an $R$-submodule of $G$, for if $y \in B$, then $m y \in R X$ for some $0 \neq m \in Z$ and $m R y \subseteq R X \subseteq B$. In fact, $B$ is an $R$-pure submodule of $G$, since if $r=n u \in R$ with $n \in Z$ and $u$ a unit of $R$, then $r G \cap B=n G \cap B=n B=r B$. Consequently, $B$ is an $R$-summand of $G$ with $R$-rank $B=1$. As a consequence of the argument given in Fuchs [3, p. 114], $B \simeq R \otimes_{Z} A$ since $G \simeq \sum_{i=1}^{k} \bigoplus\left(R \otimes_{Z} C\right)$. Write $\langle X\rangle_{*}$ for the pure subgroup of $G$ generated by $X$.

Now let $X$ and $Y$ be two pure rank 1 subgroups of $G$. Then $\langle R X\rangle_{*}$ $\simeq\left(R \otimes_{Z} A\right) \simeq\langle R Y\rangle_{*}:\langle R X\rangle_{*}$ and $\langle R Y\rangle_{*}$ are summands of $G$; and $R \otimes_{Z} A$ is strongly homogeneous so there is an automorphism $g$ of $G$ with $g(X)=Y$.

$(\Rightarrow)$ We first prove that $G$ is irreducible (i.e., if $P$ is a pure fully invariant subgroup of $G$, then $P=0$ or $G$ ). Let $0 \neq P$ be a pure fully invariant subgroup of $G ; X$ a pure rank 1 subgroup of $P$; and $Y$ a pure rank 1 subgroup of $G$. There is an automorphism $g$ of $G$ with $Y=g(X) \subseteq g(P)$ $\subseteq P$. Therefore, $P=G$. As a consequence of Reid [6], there is $0 \neq m \in Z$ with $m G \subseteq G_{1} \oplus G_{2} \oplus \cdots \oplus G_{k} \subseteq G$, where $Q \otimes_{Z} \operatorname{End}\left(G_{i}\right)$ is a division algebra with $Q$-dimension equal to rank $G_{i}$ and there are monomorphisms $f_{i j}$ : $G_{i} \rightarrow G_{j}$ with $G_{j} /$ image $f_{i j}$ bounded for all $1 \leqslant i, j \leqslant k$. Since $m\left\langle G_{i}\right\rangle_{*} \subseteq G_{i}$ $\subseteq\left\langle G_{i}\right\rangle_{*}$, for each $i$, it is sufficient to assume that each $G_{i}$ is pure in $G$.

Let $X_{i}$ and $X_{j}$ be pure rank 1 subgroups of $G_{i}$ and $G_{j}$, respectively. There is an automorphism $g$ of $G$ with $g\left(X_{i}\right)=X_{j}$. For each $1 \leqslant l \leqslant k$, let $\Pi_{l}: G \rightarrow G_{l}$ be a quasi-projection (i.e., multiplication by $m$ followed by a projection onto $\left.G_{l}\right)$. Define $g_{l}$ to be $\Pi_{l} g$, restricted to $G_{i}$. If $l \neq j$ then $g_{l}\left(X_{i}\right)=0 ; f_{l i} g_{l} \in$ $\operatorname{End}\left(G_{i}\right) ; f_{l i} g_{l}=0$ since $Q \otimes_{Z} \operatorname{End}\left(G_{i}\right)$ is a division algebra and $f_{l i} g_{l}\left(X_{i}\right)=0$; and $g_{l}=0$ since $f_{l i}$ is a monomorphism. But

$$
\begin{aligned}
m g\left(G_{i}\right) & =\Pi_{1} g\left(G_{i}\right)+\cdots+\Pi_{k} g\left(G_{i}\right) \\
& =g_{1}\left(G_{i}\right)+\cdots+g_{k}\left(G_{j}\right)=g_{j}\left(G_{i}\right) \subseteq G_{j}
\end{aligned}
$$

so that $g\left(G_{i}\right) \subseteq G_{j}$ since $G_{j}$ is pure in $G$. Since $g$ is an automorphism of $G$, it follows that $g\left(G_{i}\right)=G_{j}$. 
In particular, $G_{i} \simeq G_{j}$ for all $1 \leqslant i, j \leqslant k$ and each $G_{i}$ is strongly homogeneous. Let $A=G_{1}, R=\operatorname{End}(A)$, and $X$ a pure rank 1 subgroup of $A$. Define $\delta: R \otimes_{Z} X \rightarrow A$ by $\delta(r \otimes x)=r x$. Since $A$ is strongly homogeneous, $\delta$ is an epimorphism. But

$$
\operatorname{rank}(A)=Q \text {-dimension } Q \otimes_{Z} \operatorname{End}\left(G_{1}\right)=\operatorname{rank}(R)=\operatorname{rank}\left(R \otimes_{Z} X\right)
$$

so that $\delta$ is an isomorphism.

Next, we prove that if $0 \neq r \in R$, then $r$ is an integral multiple of a unit in $R$. Let $X$ be a pure rank 1 subgroup of $A$ such that $Y=\langle r(X)\rangle_{*}$ is a pure rank 1 subgroup of $A$. Since $A$ is strongly homogeneous, there is a unit $u \in R$ with $u(X)=Y$. The restriction of $r$ and $u$ to $X$ are nonzero elements of the rank 1 group $\operatorname{Hom}(X, Y)$ so there exist relatively prime integers $c$ and $d$ with $c u(x)=d r(x)$ for all $x \in X$. Then $d r(X)=c u(X)=c Y$, and it follows that $d Y=Y$. Since any two pure rank 1 subgroups of $A$ are isomorphic, $d A=A$; $u / d$ is an automorphism of $A$ and $(c(u / d)-r)(X)=0$. But $Q \otimes_{Z} R$ is a division algebra so $c(u / d)=r$ with $u / d$ a unit of $R$ and $c \in Z$ as desired.

Since $R=\operatorname{End}(A), R \otimes_{Z} X \simeq A$ for a rank 1 group $X$, and $Q \otimes_{Z} R$ is a division algebra, it follows that $R \simeq \operatorname{End}\left(R^{+}\right)$. Embed $R^{+}$in $Q \otimes_{Z} R$ by $r \rightarrow 1 \otimes r$ so that $R=\left\{\alpha \in Q \otimes_{Z} R \mid \alpha R^{+} \subseteq R^{+}\right\}$. It follows that $R$ is commutative and that $R$ is a principal ideal domain.

Finally, $m G \subseteq G_{1} \oplus \cdots \oplus G_{k} \subseteq G$; each $G_{i}$ is isomorphic to $A$; $\operatorname{End}(A)$ is a principal ideal domain; and since $G$ is strongly homogeneous the map $\operatorname{Hom}(A, G) \otimes_{Z} A \rightarrow G$ is an epimorphism. Therefore, $G$ is isomorphic to a finite direct sum of copies of $A$ (Arnold and Lady [1]). Since $A \simeq R \otimes_{Z} X$, for a pure rank 1 subgroup $X$ of $A, G \simeq R \otimes_{Z} H$ where $H=\sum_{i=1}^{k} \oplus X$, completing the proof.

The following corollary summarizes some consequences of the proof of Theorem 1.

COROLlaRY 2. Let $G$ be a strongly homogeneous torsion free abelian group of finite rank.

(a) $G \simeq \sum_{i=1}^{k} \bigoplus A$ where $A$ is strongly homogeneous and strongly indecomposable (i.e. $m A \subseteq A_{1} \oplus A_{2} \subseteq A$ for some $0 \neq m \in Z$ implies that $A_{1}=0$ or $A_{2}=0$ ).

(b) $G$ is indecomposable iff $G$ is strongly indecomposable.

(c) If $B$ is a direct summand of $G$ then $B \simeq \sum_{i=1}^{l} \oplus A$.

(d) If $H$ is another strongly homogeneous torsion free abelian group of finite rank, then $G \simeq H$ iff rank $G=\operatorname{rank} H, G$ and $H$ have isomorphic pure rank 1 subgroups and the center of $\operatorname{End}(G)$ is isomorphic to the center of $\operatorname{End}(H)$.

Proof. A consequence of the following observations:

(i) $G \simeq \sum_{i=1}^{k} \bigoplus A$, where $A$ is strongly homogeneous and $Q \otimes_{Z} \operatorname{End}(A)$ is a field (thus $A$ is strongly indecomposable);

(ii) $\operatorname{End}(A)$ is a principal ideal domain so that any summand of $G$ is isomorphic to a direct sum of copies of $A$ (Arnold and Lady [1]); $A$ $\simeq \operatorname{End}(A) \otimes_{Z} X$, where $X$ is a pure rank 1 subgroup of $A$; and the center of $\operatorname{End}(G)$ is isomorphic to $\operatorname{End}(A)$.

Two finite rank torsion free abelian groups $G$ and $H$ are quasi-isomorphic if there is a monomorphism $f: G \rightarrow H$ such that $H$ /image $f$ is bounded. 
Proposition 3. Suppose that $G$ and $H$ are two strongly homogeneous torsion free abelian groups of finite rank. Then $G$ and $H$ are quasi-isomorphic iff $G$ and $H$ are isomorphic.

Proof. Write $G=\sum_{i=1}^{k} \bigoplus A$ and $H=\sum_{i=1}^{l} \oplus C$ where $A$ and $C$ are strongly homogeneous and strongly indecomposable. If $G$ and $H$ are quasiisomorphic, then rank $G=\operatorname{rank} H$ and the groups $G$ and $H$ have isomorphic pure rank 1 subgroups. In view of Corollary 2(d), it is sufficient to prove that $\operatorname{End}(A) \simeq \operatorname{End}(C)$. Since $A$ and $C$ are strongly indecomposable, the KrullSchmidt theorem for quasi-decompositions (e.g., see Reid [6]) guarantees that $A$ and $C$ are quasi-isomorphic.

Let $m$ be the least positive integer such that there is a monomorphism $\phi$ : $A \rightarrow C$ with $m C \subseteq \phi(A)=B \subseteq C$. Recall that every endomorphism of $A$ (or $C$ ) is an integral multiple of an automorphism.

Suppose that $f$ is an automorphism of $B$, embed $C$ in $Q \otimes_{Z} C$ so that $Q B=Q \otimes_{Z} C$, extend $f$ to $Q \otimes_{Z} C$, and let $n$ be the least positive integer with $n f(C) \subseteq C$. Such an $n$ exists since $m f(C)=f(m C) \in f(B)=B \subseteq C$. Then $n f=k u$ for some $k \in Z, u$ an automorphism of $C$, and $p C \neq C$ for all primes $p$ dividing $k$. If $d=$ g.c.d. $(n, k)$ then $(n / d) f(C)=(k / d) u(C) \subseteq C$ so that, by the minimality of $n, d=1$. Thus $n B=n f(B)=k u(B) \subseteq k C$. Since g.c.d. $(n, k)=1, m C \subseteq B \subseteq k C$. By the minimality of $m, k=1$, since otherwise there is a monomorphism $\phi: A \rightarrow C$ with $l C \subseteq \phi(A) \subseteq C$ and $l<m$. Therefore, $n f=u, u(B)=n f(B)=n B$, and $B=u^{-1} u(B)=n u^{-1}(B) \subseteq n C$. Again by the minimality of $m, n=1$.

Every endomorphism of $B$ is an integral multiple of an automorphism of $B$ so, by the preceding remarks, there is a homomorphism $\theta: \operatorname{End}(B) \rightarrow \operatorname{End}(C)$ given by lifting endomorphisms of $B$ to $Q \otimes_{Z} C$ and restricting to $C$.

It is now sufficient to prove that $\theta$ is onto. Let $u$ be an automorphism of $C$ and $n$ the least positive integer with $n u(B) \subseteq B$. There is such an $n$ since $m u(B) \subseteq m C \subseteq B$. Write $n u=k f$ for $k \in Z$ and $f$ an automorphism of $B$. By the minimality of $n$, g.c.d. $(k, n)=1$. Therefore, $k B=k f(B)=n u(B)$ $\subseteq n C$. It follows that $m C \subseteq B \subseteq n C$ and that $n=1$ by the minimality of $m$. Thus $u=k f$ and $\theta$ is onto, as desired.

COROLlaRy 4. Let $\delta$ be the class of finite direct sums of strongly homogeneous torsion free abelian groups of finite rank.

(a) If $G$ is in $\mathcal{S}$ and $B$ is a summand of $G$ then $B$ is in $S$.

(b) Every $G$ in $\mathcal{S}$ has the Krull-Schmidt property (i.e. any two decompositions of $G$ into direct sums of indecomposable groups are equivalent).

Proof. A consequence of Corollary 2, Proposition 3, and a result of Arnold and Lady [1]. Briefly, assume that $B$ is indecomposable, write $G=\Sigma \oplus A_{i}$ where each $A_{i}$ is strongly indecomposable and strongly homogeneous, let $A=\Sigma \oplus\left\{A_{i} \mid A_{i} \simeq A_{1}\right\}$ and $K=\Sigma \oplus\left\{A_{i} \mid A_{i} \not A_{1}\right\}$ so that $G=A \oplus K$ $=B \oplus C$. Now no quasi-summand of $A$ is quasi-isomorphic to any quasisummand of $K$ since $A_{i}$ and $A_{j}$ are quasi-isomorphic iff $A_{i} \simeq A_{j}$. By Arnold and Lady [1], $B=B_{1} \oplus B_{2}, C=C_{1} \oplus C_{2}$ and $G=B_{1} \oplus C_{1} \oplus K=A \oplus$ $B_{2} \oplus C_{2}$. Therefore, either $B=B_{1} \simeq A_{1}$ or $B=B_{2}, A \simeq C_{1}, H=A \oplus K$ $=C_{1} \oplus\left(C_{2} \oplus B\right)$ and $K \simeq C_{2} \oplus B$. By induction, $B$ is strongly homogeneous. 
For the following corollaries we assume fundamental properties of subrings of algebraic number fields as, for example, in Kaplansky [4] or Samuel [8].

Proposition 5. Let $R$ be an integral domain such that the quotient field $K$ of $R$ is an algebraic number field. Then every element of $R$ is an integral multiple of a unit in $R$ iff $R=\cap\left\{J_{P} \mid P \in S\right\}$ where $J$ is the ring of algebraic integers of $K, S$ is a set of prime ideals of $J$, and if $p$ is a rational prime with $p J=P_{1}^{e_{1}} P_{2}^{e_{2}} \cdots P_{n}^{e_{n}}$, a product of powers of distinct prime ideals of $J$, then at most one $P_{i} \in S$ and $P_{i} \in S$ implies that $e_{i}=1$.

Proof. $(\Leftarrow)$ If $p$ is a rational prime with $p R \neq R$, then $p J_{P} \neq J_{P}$ for some $P \in S$. Furthermore, $p J_{P}=P J_{P}$ and $p J_{P^{\prime}}=J_{P^{\prime}}$ for all $P^{\prime} \in S \backslash\{P\}$. Therefore, $p R=p J_{P} \cap R=P J_{P} \cap R$ and the canonical map $R / p R \rightarrow J_{P} / p J_{P}$ is a monomorphism. Thus, $R / p R$ is a field; every prime ideal $R$ is of the form $p R$ for a rational prime $p$; and every ideal $I$ of $R$ can be written as $n R$ for some $n \in Z$ (since $R$ is Dedekind, every ideal is a product of prime ideals). Consequently, every element of $R$ is an integral multiple of a unit of $R$.

$\Leftrightarrow$ Since $R$ is a principal ideal domain, $R$ is integrally closed in $K$. Consequently, $J \subseteq R$. But $\{p R \mid p$ is a rational prime and $p R \neq R\}$ is precisely the set of prime ideals of $R$. Each $R_{p R}=J_{P}$ for some prime ideal $P$ of $J$ so that $P J_{P}=p J_{P}$ and $R / p R=J_{P} / p J_{P}$ is a field. Therefore, if $p J$ $=P_{1}^{e_{1}} P_{2}^{e_{2}} \cdots P_{n}^{e_{n}}$, then $P=P_{i}$ for exactly one $i$ and $e_{i}=1$ since $J_{P} / p J_{P}$ $\simeq J_{P_{i}} / P_{i}^{e_{i}} J_{P_{i}}$ is a field.

Corollary 6. Suppose that $G$ is an indecomposable torsion free abelian group of finite rank. Then $G$ is strongly homogeneous with a pure rank 1 subgroup isomorphic to a subring of $Q$ iff $G$ is isomorphic to the additive group of a ring $R$ satisfying the condition of Proposition 5.

Proof. Since $G$ is indecomposable, $G \simeq R \otimes_{Z} H$ for some rank 1 group $H$ and some $R$ such that every element of $R$ is an integral multiple of a unit of $R$ (Theorem 1).

Since $G$ is strongly homogeneous and $H$ is isomorphic to a pure rank 1 subgroup of $G, H$ must be isomorphic to a subring of $Q$. As in the proof of Theorem $1, R \simeq \operatorname{End}(G)$ so that $H$ is isomorphic to a subring of $R$ (namely $Q \cap R$ ). Therefore $R \otimes_{Z} H$ is isomorphic, as a ring, to $R$ and the proof is complete.

COROLlary 7. Let $G$ be a torsion free abelian group of finite rank.

(a) (Harrison) $G$ is p-special iff $G \simeq J_{P}$, where $J$ is the ring of algebraic integers of an algebraic number field, $p J=P_{1} P_{2}^{e_{2}} \ldots P_{n}^{e_{n}}$ a product of powers of distinct prime ideals of $J, P=P_{1}$, and $J / P \simeq Z / p Z$.

(b) (Richman [7]) $G$ is special iff $G=\bigcap_{P \in S} J_{P}$ where $J$ is the ring of algebraic integers of an algebraic number field; if $p J=P_{1}^{e_{1}} P_{2}^{e_{2}} \cdots P_{n}^{e_{n}}$, then at most one $P_{i} \in S$, and if $P_{i} \in S$ then $e_{i}=1$ and $J / P_{i} \simeq Z / p Z$.

(c) (Arnold, Vinsonhaler and Wickless [2]) If rank $G=2$ then $G$ is strongly homogeneous iff either $G \simeq A \oplus A$ or (i) $Q \otimes_{Z} \operatorname{End}(G)=Q(\vee n)$ for some square free integer $N$; (ii) $Z / p Z$ - dimension of $G / p G \leqslant 1$ for all odd primes $p$ such that $N$ is a quadratic residue $\bmod p ; Z / 2 Z-$ dimension of $G / 2 G \leqslant 1$ if $N \equiv 1(\bmod 8) ; 2 G=G$ if $N \equiv 2$ or $3(\bmod 4) ;$ and $N G=G$. 
Proof. (a), (b) Apply Corollaries 5 and 6 noting that if $G$ is strongly homogeneous and $Z / p Z$ - dimension $G / p G=1$ for some rational prime $p$ then $G$ must be indecomposable.

(c) Follows from Theorem 1, Corollary 5, Corollary 6 and the following: let $p$ be a rational prime; then $p$ odd and $N$ a quadratic residue mod $p$ imply that $p$ splits in $Q(\sqrt{ } N) ; p$ an odd divisor of $N$ implies that $p$ ramifies in $Q(\vee N)$; if $N \equiv 1(\bmod 8)$, then 2 splits in $Q(\sqrt{ } N)$; and if $N \equiv 2$ or $3(\bmod$ 4), then 2 ramifies in $Q(\vee N)$ (e.g., see Samuel [8]).

\section{REFERENCES}

1. D. M. Arnold and E. L. Lady, Endomorphism rings and direct sums of torsion free abelian groups, Trans. Amer. Math. Soc. 211 (1975), 225-237.

2. D. M. Arnold, C. I. Vinsonhaler and W. J. Wickless, Quasi-pure projective and injective torsion free abelian groups of rank 2, Rocky Mountain J. Math. (to appear).

3. L. Fuchs, Infinite abelian groups. Vol. II, Academic Press, New York, 1973.

4. I. Kaplansky, Commutative rings, Allyn and Bacon, Boston, Mass., 1970. MR40 \#7234.

5. C. E. Murley, The classification of certain classes of torsion free Abelian groups of finite rank, Pacific J. Math. 40(1972), 647-665. MR48 \#441.

6. J. D. Reid, On the ring of quasi-endomorphisms of a torsion-free group, Topics in Abelian Groups (Proc. Sympos. New Mexico State Univ., 1962), Scott, Foresman, Chicago, Ill., 1963, pp. 51-68. MR30 \# 158.

7. F. Richman, $A$ class of rank-2 torsion free groups, Studies on Abelian Groups (Sympos., Montpellier, 1967), Springer, Berlin, 1968, pp. 327-333. MR39 \# 5695.

8. P. Samuel, Theorie algébrique des nombres, Hermann, Paris, 1967; English transl., HoughtonMifflin, Boston, Mass., 1970. MR35 \#6643; 42 \#177.

Department of Mathematics, New Mexico State University, Las Cruces, New Mexico 88003 